\title{
La Responsabilidad Social Corporativa en
} las empresas y su relación con los públicos de interés Los casos de Unilever y Nestlé

corporate Social

Responsibility in Companies and its

Relation to Stakeholders

The Cases of Unilever and Nestlé

Estrella Barrio

Universidad Autónoma de Barcelona Estrella.barrio@uab.cats

Ana M. a Enrique

Universidad Autónoma de Barcelona

Anamaría.enrique@uab.cat
Barrio, E., y Enrique, A. M. ${ }^{a}$, (2015). La Responsabilidad Social Corporativa en las empresas y su relación con los públicos de interés: los casos de Unilever y Nestlé. Revista Internacional de Investigación en Comunicación aDResearch ESIC. No $11 \mathrm{Vol} 11$.

Primer semestre, enero-junio 2015. Págs. 26 a 39 DOI: 11.7263/ADRESIC.011.002 
Las últimas décadas han sido testigo del auge de la implementación de la Responsabilidad Social Corporativa (RSC) por parte de las empresas y de su estudio desde la Academia. Algunas investigaciones muestran el creciente interés de las empresas por comunicar sus acciones de RSC especialmente a través de las webs corporativas. Esta investigación, basada en la metodología del estudio de caso, se centra en analizar cómo Nestlé España y Unilever España dan a conocer a través de sus webs la RSC que implementan, estableciendo una comparación entre ambas, así como la identificación de los principales stakeholders a los que dirigen sus acciones de RSC y la determinación de la temática de las acciones para cada stakeholder identificado. En síntesis podemos concluir que los stakeholders primordiales son los clientes, los proveedores, los traba-

Clasificación JEL: M140

Key words: Responsabilidad Social Corporativa, empresas, comunicación, stakeholders, casos. jadores y la sociedad en general. En cuanto a la temática de las acciones de RSC implementadas para cada uno de sus stakeholders sabemos que, cuando comunican sus acciones de RSC dirigidas a los clientes, se centran en productos saludables y nutritivos, hablando de los beneficios sobre la salud que conlleva una dieta equilibrada, lo cual, se puede conseguir consumiendo sus productos. La temática dirigida al empleado se centra, por una parte, en temas relacionados con las condiciones de la plantilla, y por otra parte, en la igualdad de oportunidades. Respecto a los proveedores, el tema que más se trata es el desarrollo rural de las regiones donde se cultivan las materias primas que utilizan. Y por último, la sociedad en general, para las que realizan acciones centradas en el cuidado del medioambiente y en la ayuda alimentaria.

\section{ABSTRACT}

\section{JEL Classification: M140}

Palabras clave:

Corporate Social Responsibility, companies, communication, stakeholders, cases
The previous decades have seen the boom of the implementation of Corporate Social Responsibility by companies and the study of it by the Academy. Some investigations show a growing interest by the companies to report on actions of CSR especially through corporate webs. This investigation, based on the methodology case study, focuses on analysing how Nestlé España and Unilever España inform through their webs the CSR they implement, establishing a comparison between the two, as well as identifying the main stakeholders who the actions of CSR are orientated towards and determining the theme of the actions for each stakeholder identified. In synthesis we can conclude that the primary stakeholders are the customers, the suppliers the employees and society in general. In regard to the subject of the implemented actions of CSR for each stakeholder we know that when actions of CSR orientated towards the clients are reported, they focus on healthy and nutritious products, commenting on the healthy benefits that a balanced diet entails which can be obtained consuming their products. The subject orientated towards the employees focuses on the one hand in matters related to staff working conditions and on the other to equal opportunities. Regarding suppliers the most apparent is the rural development of the regions where the raw materials they use are cultivated. Finally with respect to the society in general they carry out actions focusing on the environment and food aid. 


\section{Introducción}

En la actualidad el debate que se crea entorno al concepto de Responsabilidad Social Corporativa (RSC) trata de abordar cuál es el impacto que esta actividad está teniendo en las empresas y en la sociedad en general. Son numerosos los estudios que se han realizado y se realizan sobre las acciones de RSC que implementan las empresas y nos ha parecido muy interesante reflexionar acerca de este fenómeno desde la perspectiva comunicativa. Algunos de los estudios, que posteriormente referenciaremos en el marco teórico, nos indican que las empresas están otorgando especial relevancia al hecho de comunicar sus acciones de RSC, a pesar de algunas reticencias aún vigentes en el entramado empresarial debido al posible escepticismo que puede surgir entre los stakeholders de la organización por considerar esta actividad -comunicar la RSC- como oportunista. En este artículo nos interesa centrar la discusión de la RSC en la identificación o tipificación de aquellos stakeholders a los que la empresa dirige sus acciones de RSC y cuál es la naturaleza de éstas. Tanto la identificación de los stakeholders como la temática de las acciones son aspectos que se derivan de la comunicación intencional de la RSC por parte de las empresas. Para delimitar el estudio hemos considerado pertinente estudiar los casos de dos empresas relevantes en el sector de la alimentación como son Unilever y Nestlé. Las preguntas que nos hacemos son las siguientes:

Q1: En la comunicación de la RSC que realizan Unilever y Nestlé en España través de sus webs corporativas, ¿cuáles son los principales stakeholders a quién van dirigidas las acciones de RSC?

Q2: En la comunicación de la RSC que realizan Unilever y Nestlé en España través de sus webs corporativas, ¿cuál es la temática de las acciones de RSC para cada uno de los stakeholders?

\section{Marco Teórico}

\subsection{Concepto de Responsabilidad Social Corporativa}

En lo referente al concepto de RSC, se ha de apuntar que no existe una definición unánime del concepto de RSC, aunque como veremos más adelante sí que se observan ciertos elementos coincidentes en las definiciones que a continuación apuntaremos. En la actualidad, podemos decir que nos encontramos con dos enfoques distintos de entender cómo se desarrolla la RSC en las organizaciones. Por un lado, hablaríamos de la RSC basada en el Modelo Shareholder, por el cual el único objetivo de la empresa es el de crear valor para el accionista; y por otro lado, el Modelo Stakeholder o Plural, por el cual el objetivo de una empresa consiste en crear valor para el conjunto de los grupos de interés. Este segundo enfoque nos habla de la importancia de no pensar sólo en el valor positivo que puede ofrecer únicamente a los accionistas si nos comportamos con responsabilidad y ética, sino que nos está planteando la necesidad y los beneficios de hacerlo bien, de que la empresa actúe como un buen ciudadano ante sus stakeholders y establezca una relación mutua con el resto de públicos de interés de la organización, con proveedores, clientes, empleados y comunidades locales, entre otros.

Siguiendo este segundo enfoque, son diversos los autores que han definido el concepto de RSC, y más diversas aún, son las definiciones que plantean. Sin embargo, podemos atisbar una serie de criterios que parecen repetirse. Van Marrewijk (2003) y Waldan et al. (2006) señalan la relación de la empresa con sus grupos de interés, mientras que autores como Jones (1980), 
Smith (2003) y Maignan y Ferrell (2004), van más allá haciendo especial hincapié en una obligación de la empresa hacia sus grupos de interés. Otros autores destacan en sus definiciones aquellas acciones que realiza la empresa que van más allá de los intereses económicos de la organización (Davis, 1960), es decir, que buscan un bienestar socioeconómico general (Frederick, 1960). La Comisión Europea (2001 y 2011), De la Cuesta y Valor (2003), van Marrewijk (2003), Watson y MacKay (2003) y Panwar et al. (2006) destacan las preocupaciones sociales y medioambientales por parte de las empresas en el desarrollo de sus actividades de negocio. Otro criterio que se repite a la hora de definir RSC, es la responsabilidad de las empresas por los impactos negativos en la sociedad derivados de sus actividades, es decir, que las empresas se comporten de manera no dañina para la sociedad (Castillo, 1986; Andrews, 1987; Mohr Webb y Harris, 2001; Basil y Weber, 2006; Comisión Europea, 2011). En esta misma línea, otros autores destacan el deber de las empresas a actuar de manera beneficiosa para la sociedad, maximizando su impacto positivo en la sociedad (Frederick, 1983; Andrews, 1987; Kok, van der Wiele, McKenna y Brown, 2001; Basil y Weber, 2006; Comisión Europea, 2011).

Por consiguiente, se puede remarcar que la RSC va más allá de la propia acción social que implementen las empresas o la filantropía de sus dirigentes. La RSC no es únicamente financiar la construcción de un pozo en una remota aldea de un país subdesarrollado, plantar árboles en la selva amazónica o patrocinar actos deportivos y/o culturales para de este modo, acallar las voces que acusan a las organizaciones por no respetar los derechos humanos o por contribuir al deterioro del medioambiente. La RSC debe ser la consecuencia de un compromiso adoptado con todos sus grupos de interés en materia económica, social y medioambiental, con el objetivo de responsabilizarse de las consecuencias e impactos que derivan de sus acciones y maximizar la creación de valor compartido para todo el conjunto de stakeholders. La RSC no se queda en la superficie de las organizaciones, si no que se sitúa en lo más profundo de éstas, trabajando siempre de acorde con la visión, misión, valores y proyecto empresarial de la organización.

A la hora de teorizar sobre las dimensiones del concepto de RSC, también existen diferentes propuestas. Una de las primeras concepciones es la formulada por Carroll (1979), quien define la RSC como un concepto multidimensional que incluye aspectos económicos, legales, éticos y filantrópicos. Aunque la perspectiva más actual para trabajar las dimensiones del concepto de RSC, es la de los diferentes grupos de interés. Desde esta perspectiva, Clarkson (1995), considera que se debe hablar de «preocupaciones de los grupos de interés» en lugar de «preocupaciones legales». En esta misma línea, Decker (2004) distingue cuatro dimensiones de la RSC: la sociedad, el mercado (clientes, proveedores y competidores), el entorno de trabajo (los empleados) y el medioambiente.

\subsection{Comunicación de la RSC}

En los últimos años, hemos vivido un aumento de la comunicación de RSC que realizan las empresas, y es que, parece que está habiendo un creciente interés de la RSC desde las áreas de marketing y comunicación, convirtiendo, tal y como menciona Podnar (2008) la comunicación de la RSC en algo importante y relevante para la compañía. 
Diferentes estudios llevados a cabo en los últimos años muestran el auge que está viviendo la comunicación de la RSC por parte de las corporaciones.

El primer ejemplo lo encontramos en Mögele y Tropp (2010), quienes realizan un estudio longitudinal en Alemania, analizando los anuncios de RSC publicados en tres revistas alemanas durante los años 2002 a 2007. Los resultados del estudio han demostrado un aumento de la publicidad dedicada a las acciones de RSC por parte de las empresas.

Un segundo ejemplo es el estudio de Arvidsson (2010), donde también se muestra la creciente importancia otorgada por parte de las compañías a comunicar sus acciones de RSC. En este caso, se realizaron entrevistas en profundidad y cuestionarios a directivos de 27 de las 30 empresas que formaban el OMX Stockholm 30. Los resultados de la investigación, concluyeron la existencia de una tendencia creciente en focalizar la RSC desde la comunicación corporativa. De hecho, consideran la RSC como uno de los ítems no relacionados con información financiera más importantes a comunicar. El argumento es que los escándalos mediáticos han aumentado el interés de las empresas por incrementar las políticas estratégicas de RSC, y por consiguiente, ha habido un aumento de la información sobre RSC en la comunicación corporativa de las empresas, la cual empezó siendo reactiva a los escándalos corporativos. No obstante, el estudio muestra que la tendencia ha cambiado para pasar a ser proactiva, centralizada en prevenir la legitimidad de la empresa.

Por su parte, Podnar (2008) explica que Basil y Erlandson llevaron a cabo una investigación similar en Canadá, realizando un estudio longitud, de 2003 a 2006, en el que analizaron la apa- rición de información sobre RSC en las webs corporativas de 159 empresas canadienses. Los resultados revelaron que únicamente el 27\% de las empresas incluía información de RSC en sus webs corporativas en el año 2003, frente a un $67 \%$ en 2006, lo que demuestra el creciente aumento de la comunicación de la RSC a través de las webs corporativas. El estudio añade que las empresas más exitosas tienen una mayor información de RSC en sus webs.

En España, nos encontramos ante la misma situación. Cappriotti y Moreno (2007) presentaron un análisis de la información sobre las acciones de RSC que aparecían en las webs de las empresas que formaban parte del IBEX35, demostrando la importancia que otorgan las compañías a comunicar su RSC a través de sus webs corporativas, ya que el 100\% de las empresas analizadas difundían sus acciones de RSC a través de sus propias webs, lo cual muestra la importancia actual otorgada a utilizar las páginas web como canal para comunicar sus acciones de RSC. De hecho, según un estudio realizado por MediaResponsables y Dircom (2010), la herramienta que, los profesionales del sector de la comunicación y la RSC en España, consideran más útil para comunicar sus actividades de RSC son las webs y portales corporativos, mientras que la publicidad y el marketing directo son los peores evaluados.

En cuanto al contenido de las comunicaciones, destaca el estudio llevado a cabo por Illia, Rodríguez, González y Romenti (2010), en el que analizan la comunicación de RSC que realizan 250 empresas europeas, siendo la muestra de 42 empresas por país (España, Italia, Francia, Dinamarca, Suecia y Reino Unido). Para ello, analizan el contenido de la información sobre RSC que las empresas comunican a través de: las 
webs corporativas, los informes sociales, la publicidad social, los blogs corporativos y la presencia en web 2.0. Los resultados expuestos en la tabla 1, indican que existe un compromiso generalizado para comunicar la RSC de los procesos centrales del negocio, ya que las empresas consideran que los stakeholders conocen el impacto social y medioambiental de sus actividades. Por el contrario, las compañías se muestran más cautelosas a la hora de publicar actividades no conectadas con su negocio, como las actividades caritativas (Illia, Rodríguez, González y Romenti, 2010).

De las investigaciones descritas anteriormente se puede extraer que, se puede diferenciar en- tre aquellas organizaciones que realizan una comunicación reactiva como respuesta a la presión pública; y aquellas que realizan una comunicación proactiva para conseguir o mantener su legitimidad en la sociedad. En los últimos años, la tendencia es realizar una comunicación proactiva de la RSC. Para ello, las empresas utilizan diferentes herramientas, entre las que destacan las webs y portales corporativos, consideradas por los profesionales del sector de la comunicación y la RSC en España, como una de las herramientas más efectivas para comunicar las actividades de RSC. Por otra parte, existen diferentes contenidos en la información de RSC que comunican las organizaciones, aunque parece que los conte-

\section{Tabla 1 - Contenido información RSC en webs}

\begin{tabular}{|c|c|}
\hline Contenido & Porcentaje de empresas que lo comunican \\
\hline $\begin{array}{l}\text { Informaciones que involucran a la } \\
\text { comunidad }\end{array}$ & $\begin{array}{l}\text { El } 65 \% \text { describe sus proyectos de colaboración con comunidades locales, } \\
\text { el } 47 \% \text { las políticas para jóvenes graduados y el } 66 \% \text { acciones de patrocinio, } \\
\text { donaciones, fundaciones y eventos. }\end{array}$ \\
\hline $\begin{array}{l}\text { Informaciones sobre temas que afectan } \\
\text { al mercado }\end{array}$ & $\begin{array}{l}\text { El } 66 \% \text { incluye información sobre l+D de productos sociales, el } 65 \% \text { en seguridad } \\
\text { en producción y distribución, el } 70 \% \text { en atención al cliente y el } 66 \% \text { en estándares } \\
\text { de calidad de producción. }\end{array}$ \\
\hline $\begin{array}{l}\text { Informaciones sobre temas éticos } \\
\text { y de gobernanza }\end{array}$ & $\begin{array}{l}\text { El } 65 \% \text { informa sobre su conducta ética, mientras que sólo el 37\% informa sobre } \\
\text { cuestiones de comercio justo. }\end{array}$ \\
\hline $\begin{array}{l}\text { Informaciones sobre temas que afectan } \\
\text { al desarrollo de la economía local }\end{array}$ & $\begin{array}{l}\text { El 31\% informa sobre proyectos para mejorar el desarrollo económico, el 51\% } \\
\text { proyectos para la educación y el 39\% proyectos para crear puestos de trabajo }\end{array}$ \\
\hline $\begin{array}{l}\text { Informaciones sobre asuntos de } \\
\text { medioambiente }\end{array}$ & $\begin{array}{l}\text { El } 77 \% \text { informa sobre su contribución al desarrollo sostenible, el } 69 \% \text { sobre temas } \\
\text { de contaminación y el } 64 \% \text { trata de sensibilizar a la gente sobre cuestiones } \\
\text { medioambientales. }\end{array}$ \\
\hline Informaciones sobre el clima laboral & $\begin{array}{l}\text { El } 50 \% \text { facilita información sobre los beneficios y salarios de sus empleados, el } 69 \% \\
\text { sobre de formación y desarrollo de carrera, el } 65 \% \text { sobre igualdad de oportunidades } \\
\text { y el } 69 \% \text { sobre condiciones del lugar de trabajo. }\end{array}$ \\
\hline Informaciones sobre derechos humanos & $\begin{array}{l}\text { Rara vez comparten públicamente información sobre derechos humanos. } \\
\text { Sólo el } 43 \% \text { comunica el rechazo al trabajo infantil }\end{array}$ \\
\hline
\end{tabular}


nidos más comunes son aquellos relacionados con los procesos centrales de la propia organización.

\section{Metodología}

Se ha planteado una investigación basada en el método del estudio de caso. Este método de investigación es muy apropiado cuando el objetivo es comprender y explicar un fenómeno utilizando las fuentes de información que se requieran. En esta investigación no pretendemos buscar una representación estadística. Nuestra aspiración es alcanzar una generalización analítica en la medida en que como explica Coller (2005, p. 68) «el caso es pertinente teóricamente (es decir, relevante), se puede relacionar las conclusiones del estudio con una teoría o conjunto de ellas (...)». Estas conclusiones no se refieren a una población o universo, sino a unas teorías previas.

Los casos seleccionados han sido Nestlé España y Unilever España, dos empresas relevantes que operan en el sector de la alimentación, con una amplia gama de productos que se comercializan en este país. Las dos empresas cuentan con premios y reconocimientos de sostenibilidad. En 2013, Nestlé lideró el índice Dow Jones Sustainability del sector de la alimentación y consiguió máxima puntuación en el CDP Climate Disclosure Leadership Index y en el CDP Climate Performance Leadership Index (Nestlé, 2014). En el mismo año, Unilever fue nombrada líder por el CDP's Forests Program, lideró el Tomorrow Value's Rating sobre prácticas de sostenibilidad corporativa en el sector de la alimentación y bebidas, también ganó el premio the Best Sustainability and Stakeholder Disclosure FTSE 100, y recibió la Gold Medal for International Corporate Achievement in Sustainable Development por el World Environment Center (Unilever, 2014). Además, ambas compañías es- tán presentes en publicaciones españolas especializadas en RSC como buenos ejemplos de gestión y comunicación de RSC. A modo de ejemplo, se puede citar la presencia de las organizaciones en el anuario 2013 de Corresponsables que edita Mediarresponsable, en la publicación online Compromiso RSE y en la publicación de Mediaresponsables y DIRCOM titulada La aplicación de las herramientas de Comunicación a la RSE.

Para llevar a cabo el estudio de estos dos casos, se ha realizado un análisis de contenido de las comunicaciones de RSC que divulgan a través de sus webs corporativas. Concretamente, se ha seleccionado la información de RSC que, a fecha de 28 de noviembre de 2013, presentan Unilever España y Nestlé España en sus webs corporativas. En el caso de Unilever sólo se ha tenido en cuenta la información vinculante al sector de la alimentación, no se ha valorado la información relativa al sector de la higiene personal y limpieza del hogar. Se ha practicado este tipo de análisis, ya que, tal y como define Kerlinger (1973), el análisis de contenido es un método de estudio y análisis de comunicación de forma sistemática, objetiva y cuantitativa, con la finalidad de medir determinadas variables. En nuestro caso, las variables que se han medido son la presencia y el contenido de información de acciones de RSC relacionadas con los principales stakeholders de la organización. Los principales stakeholders detectados, se han dividido a su vez en diferentes categorías según la temática de las acciones. Estas categorías han sido elaboradas a partir de los resultados obtenidos durante la investigación, ya que se disponía de escasa información documental que permitiera elaborar alguna categorización temática previa a la propia investigación. 


\section{Resultados}

En referencia a la Q1, tal y como se puede observar en la tabla 2, en la comunicación de la RSC que realizan Unilever y Nestlé España a través de sus webs corporativas, los principales stakeholders que se han podido identificar son: los clientes, los proveedores, los empleados y la sociedad en general.

En relación a la temática de las acciones de RSC para cada uno de estos stakeholders (Q2), se puede comentar, que las acciones de RSC dirigidas a los clientes tratan sobre productos saludables y nutritivos; las acciones de RSC dirigidas a los proveedores tratan sobre el desarrollo rural de las zonas de cultivo, normalmente situadas en países en desarrollo, de los cuales las empresas obtienen sus materias primas; las acciones de RSC dirigidas a los empleados, pueden dividirse en tres grandes categorías: las condiciones laborales en la empresa, la no discriminación o igualdad de oportunidades entre los miembros de sus plantillas, y la sensibilización de los hábitos de alimentación de sus plantillas; en cuanto a la sociedad en general, la información que ofrecen, se puede dividir en acciones sociales dedicadas a la donación de alimentos, acciones sociales dedicadas a la educación alimentaria, acciones sociales dedicadas a la erradicación de la pobreza y en información medioambiental.

Con el objetivo de profundizar más en el contenido de la información expuesta para cada uno de los grupos de interés, las diferentes temáticas se han dividido en sub-temáticas. En la tabla 2, se muestra el conjunto de temáticas y sub-temáticas obtenidas a partir del análisis de contenido.

Como se puede observar en la tabla, respecto a la información de RSC sobre los clientes, ambas empresas tratan sobre nutrición, presentan- do las enfermedades derivadas de una incorrecta alimentación y los beneficios sobre la salud que conlleva una alimentación variada y equilibrada, cosa que se puede conseguir, según ellas, si se consumen sus productos, los cuales aportan unas cantidades adecuadas de nutrientes. También citan sus etiquetados nutricionales, cuya finalidad es ayudar al consumidor a llevar una dieta nutricionalmente equilibrada. Únicamente Unilever, realiza una pequeña mención sobre las acciones de motivación para que los clientes elijan productos sostenibles.

En lo relativo a la información que ofrecen sobre proveedores, en ambos casos hacen referencia al desarrollo rural de las zonas de cultivo de las cuales obtienen sus materias primas como el cacao, el té o el café. A excepción de las explotaciones lecheras de Nestlé y las granjas de gallinas de Unilever, el contenido de este apartado se centra en las acciones de apoyo y formación que las dos empresas llevan a cabo con el objetivo de fomentar una agricultura sostenible y el desarrollo de las comunidades, aportando beneficios ambientales, sociales y económicos que conllevan a un futuro mejor. Ambas empresas hacen especial hincapié en el rol de las mujeres agricultoras de estas comunidades y en cómo son ayudadas desde Nestlé y Unilever. En este apartado también se menciona el origen del aceite de palma utilizado por ambas compañías.

La información sobre los trabajadores, se centra en las condiciones laborales de la plantilla, concretamente en aspectos relacionados con el desarrollo profesional dentro de la empresa; los planes de formación para los empleados, incluyendo técnicas como el coaching y el mentoring; la conciliación de la vida laboral y familiar a través de horarios flexibles; y la seguridad laboral, ofreciendo lugares de trabajo seguros y saludables; 


\section{Tabla $\mathbf{2}$ - Grupos de interés y temáticas}

\begin{tabular}{|c|c|c|c|}
\hline Públicos & Temáticas & Sub-temáticas - NESTLÉ & Sub-temáticas - UNILEVER \\
\hline \multirow{10}{*}{ Clientes } & \multirow{10}{*}{$\begin{array}{l}\text { Productos } \\
\text { saludables y } \\
\text { nutritivos }\end{array}$} & Tipos de nutrientes & Tipos de nutrientes \\
\hline & & Cantidades adecuadas de nutrientes & Cantidades adecuadas de nutrientes \\
\hline & & $\begin{array}{l}\text { Enfermedades derivadas de una } \\
\text { inadecuada alimentación }\end{array}$ & $\begin{array}{l}\text { Enfermedades derivadas de una } \\
\text { inadecuada alimentación }\end{array}$ \\
\hline & & Salud & Salud \\
\hline & & Alimentación equilibrada/saludable & Alimentación equilibrada/saludable \\
\hline & & Etiquetado nutricional & Etiquetado nutricional \\
\hline & & $\begin{array}{l}\text { Adición de nutrientes beneficiosos } \\
\text { para la salud }\end{array}$ & $\begin{array}{l}\text { Adición de nutrientes beneficiosos } \\
\text { para la salud }\end{array}$ \\
\hline & & Profesionales de la nutrición & \\
\hline & & Fundamentos de nutrición & \\
\hline & & & $\begin{array}{l}\text { Motivar a los clientes a elegir marcas } \\
\text { sostenibles }\end{array}$ \\
\hline \multirow{11}{*}{ Proveedores } & \multirow{11}{*}{ Desarrollo rural } & Agricultura y ganadería & Agricultura y ganadería \\
\hline & & Agricultura sostenible & Agricultura sostenible \\
\hline & & Formación de agricultores & Formación de agricultores \\
\hline & & Apoyo a los agricultores & Apoyo a los agricultores \\
\hline & & Derechos humanos y laborales & Derechos humanos y laborales \\
\hline & & Tipos de materias & Tipos de materias \\
\hline & & $\begin{array}{l}\text { Las mujeres agricultoras en los países en } \\
\text { desarrollo }\end{array}$ & $\begin{array}{l}\text { Las mujeres agricultoras en los países } \\
\text { en desarrollo }\end{array}$ \\
\hline & & Futuro & Futuro \\
\hline & & & Mejora de la calidad de vida de los agricultores \\
\hline & & Desarrollo de la comunidad & \\
\hline & & Pesticidas & \\
\hline \multirow{10}{*}{ Trabajadores } & \multirow{5}{*}{$\begin{array}{l}\text { Condiciones } \\
\text { laborales }\end{array}$} & Desarrollo profesional & Desarrollo profesional \\
\hline & & Formación & Formación \\
\hline & & Conciliación vida laboral-personal & Conciliación vida laboral-personal \\
\hline & & Seguridad y salud laboral & Seguridad y salud laboral \\
\hline & & Bienestar de los trabajadores & Bienestar de los trabajadores \\
\hline & \multirow{3}{*}{$\begin{array}{l}\text { Igualdad de } \\
\text { oportunidades }\end{array}$} & Igualdad de género & Igualdad de género \\
\hline & & Discapacitados & Discapacitados \\
\hline & & & Diversidad cultural \\
\hline & $\begin{array}{l}\text { Sensibilización de } \\
\text { los hábitos de } \\
\text { alimentación }\end{array}$ & $\begin{array}{l}\text { Sensibilización de los hábitos de aliment- } \\
\text { ación de los trabajadores }\end{array}$ & $\begin{array}{l}\text { Sensibilización de los hábitos de alimentación } \\
\text { de los trabajadores }\end{array}$ \\
\hline & $\begin{array}{l}\text { Sensibilización } \\
\text { sobre sostenibilidad }\end{array}$ & & $\begin{array}{l}\text { Fomento del concepto de sostenibilidad } \\
\text { entre los trabajadores }\end{array}$ \\
\hline
\end{tabular}




\begin{tabular}{|c|c|c|c|}
\hline Públicos & Temáticas & Sub-temáticas - NESTLÉ & Sub-temáticas - UNILEVER \\
\hline \multirow{22}{*}{ Sociedad } & \multirow{4}{*}{$\begin{array}{l}\text { Acción social (asis- } \\
\text { tencia alimentaria) }\end{array}$} & Déficit nutricional - hambre & Déficit nutricional - hambre \\
\hline & & Ayuda contra el hambre & Ayuda contra el hambre \\
\hline & & Correcta alimentación & Correcta alimentación \\
\hline & & Colaboración con ONG's & Colaboración con ONG's \\
\hline & \multirow{3}{*}{$\begin{array}{l}\text { Acción social (pro- } \\
\text { gramas educativos } \\
\text { de nutrición) }\end{array}$} & Obesidad infantil & \\
\hline & & Educación nutricional & \\
\hline & & Expertos en nutrición & \\
\hline & \multirow{4}{*}{$\begin{array}{l}\text { Acción social (Er- } \\
\text { radicación de la } \\
\text { pobreza) }\end{array}$} & & $\begin{array}{l}\text { Proyectos para personas que viven en la } \\
\text { pobreza }\end{array}$ \\
\hline & & & Sensibilización de la población \\
\hline & & & Trabajo con responsables políticos \\
\hline & & & Colaboración con ONG's \\
\hline & \multirow{11}{*}{ Medioambiente } & Consumo de agua & Consumo de agua \\
\hline & & Emisiones de gases & Emisiones de gases \\
\hline & & Consumo de energía & Consumo de energía \\
\hline & & Energías renovables & Energías renovables \\
\hline & & Reciclaje & Reciclaje \\
\hline & & Reducción del material de los envases & Reducción del material de los envases \\
\hline & & Medioambiente & Medioambiente \\
\hline & & Sostenibilidad & Sostenibilidad \\
\hline & & Recursos naturales & \\
\hline & & & Reducción de trayectos de transporte \\
\hline & & & Reducción de los residuos \\
\hline
\end{tabular}

a los que Nestlé añade revisiones médicas. Todo ello con el objetivo de conseguir el bienestar de los empleados. También informan sobre la igualdad de oportunidades, a través de políticas no discriminatorias por razones de sexo, etnia o discapacidad. También ofrecen información sobre la sensibilización de sus trabajadores hacia unos hábitos de vida saludables. En el caso de Unilever, este apartado se amplía con información sobre el fomento del concepto de sostenibilidad entre la plantilla.

El último bloque, recoge la información sobre las acciones de RSC dirigidas a la sociedad en general. En este caso, se pueden diferenciar claramente dos tipos de informaciones, por un lado la información sobre acciones medioambientales, y por otro las acciones dirigidas a colectivos desfavorecidos de la sociedad, lo que se ha denominado Acción social.

En cuanto a la información sobre medioambiente, ambas empresas comunican la reducción del consumo de agua en sus procesos de producción (y agricultura en el caso de Unilever), incluyendo información sobre el tratamiento de aguas residuales a través de depuradoras. También 
mencionan las reducciones de gases de $\mathrm{CO} 2$, la reducción del consumo de energía, el uso de determinadas fuentes sostenibles, y el reciclaje y la reutilización de sus residuos, la reducción del material de los envases, así como la reducción de los trayectos de transporte y la reducción de los residuos en fábrica, oficina y post-consumo por parte de Unilever. Para comunicar todas estas acciones, ambas compañías utilizan repetidamente palabras relacionadas con el medioambiente, los recursos naturales, la sostenibilidad y el futuro.

En lo referente a la información sobre acción social, únicamente Nestlé menciona sus progr mas educativos de nutrición; y Unilever sus programas para erradicar la pobreza. En ambos casos, la temática en esta sección, se centra en la asistencia alimentaria a través de colaboraciones con entidades sin ánimo de lucro donando alimentos o dinero para ayudar a combatir el hambre y la malnutrición entre la gente más necesitada.

\section{Conclusiones}

A través de esta investigación se ha conseguido avanzar en el conocimiento e identificación de los principales stakeholders para las empresas del sector de la alimentación en España en sus comunicaciones sobre RSC. Gracias a la presente investigación, sabemos que, en la comunicación de la RSC que realizan las dos empresas analizadas, los principales stakeholders son los clientes, los proveedores, los trabajadores y la sociedad en general.

Además de esta clasificación, a partir del análisis de contenido, conocemos la temática de las acciones de RSC implementadas para cada uno de sus stakeholders, así sabemos que, cuando comunican sus acciones de RSC dirigidas a:
- Clientes, se centran en productos saludables y nutritivos, hablando de los beneficios sobre la salud que conlleva una dieta equilibrada, lo cual, se puede conseguir consumiendo sus productos.

- Empleados, se centran, por una parte, en temas relacionados con las condiciones de la plantilla, como el desarrollo profesional, la formación para empleados, la conciliación de la vida laboral y familiar y la seguridad laboral; y por otra parte, en la igualdad de oportunidades, a través de políticas no discriminatorias por razones de sexo, etnia o discapacidad.

- Proveedores, se centran en el desarrollo rural de las regiones donde se cultivan las materias primas que utilizan. Concretamente, describen las acciones de apoyo y formación que llevan a cabo con el objetivo de fomentar una agricultura sostenible y el desarrollo de las comunidades, aportando beneficios ambientales, sociales y económicos que conllevan a un futuro mejor de la comunidad. También se destaca el papel de la mujer agricultora en estas comunidades.

- Sociedad en general, este stakeholder se puede dividir en dos bloques claramente diferenciados:

- Medioambiente, se centran en la reducción del impacto de sus actividades en el medioambiente, concretamente mencionan: la reducción del consumo de agua en sus actividades, las reducciones de gases de $\mathrm{CO} 2$, la reducción del consumo de energía, el uso de determinadas fuentes sostenibles, y el reciclaje y la reutilización de 
sus residuos. Siempre utilizando palabras relacionadas con el medioambiente, los recursos naturales, la sostenibilidad y el futuro.

- Acción social, se centran en la asistencia alimentaria que realizan a través de colaboraciones con entidades sin ánimo de lucro donando alimentos o dinero para ayudar a combatir el hambre y la malnutrición entre la gente más necesitada.

Estos resultados, permiten dar un paso más en la identificación de los diferentes stakeholders que son considerados relevantes a la hora de abordar las comunicaciones de las acciones de RSC que realizan las empresas y más concretamente en el conocimiento de la propia naturaleza de las acciones llevadas a cabo con cada uno de los stakeholders.

La importancia de esta investigación, recordemos, recae en la buena reputación para la organización que el comportamiento ético de ésta provoca, ya que la hace merecedora de confianza por parte de sus grupos de interés. Las aportaciones de esta investigación suponen un paso más en el conocimiento sobre la comunicación de este valor intangible.

\section{Bibliografía}

Andrews, K.R. (1987). The concept of corporate strategy. (3rd ed.). Homewood, IL: H. Dow Jones - Irwin.

Arvidsson, S. (2010). Communication of Corporate Social Responsibility: A study of the Views of management Teams in Large Companies. Journal of Business Ethics, 96, 339-354.

Basil, D.Z. y Weber, D. (2006). Values motivation and concern for appearances: The effect of personality traits on responses to corporate social responsibility. International Journal of Nomprofit and Voluntary Sector Marketing, 11(1), 61-72.

Capriotti, P. y Moreno, A. (2007). Communicating corporate responsibility through corporate web sites in Spain. Corporate Communications: An International Journal, 12(3), 221-237.

Carroll, A.B. (1979). A three-dimensional conceptual model of corporate performance. The Academy of Management Review, 4(4), 497-505.

Castillo, A.M. (1986). La responsabilidad de la empresa en el contexto social: Su articulación, gestión y control. Doctoral thesis. Universidad de Málaga.

Clarkson, M.B.E. (1995). A stakeholder framework for analyzing and evaluating corporate social performance. The Academy of Management Review, 20(1), 92-117.
Coller, X. (2005). Estudios de casos. Centro de investigaciones Sociológicas: Madrid.

Comisión Europea. (2001). Libro Verde de la Comisión Europea para Fomentar un Marco Europeo para la Responsabilidad Social de las Empresas. Brussels.

Comisión Europea. (2011). Estrategia renovada de la UE para 2011-2014 sobre la responsabilidad social de las empresas. Brussels.

Davis, K. (1960). Can business afford to ignore social responsibilities? California Management Review, 2,(3), 70-76.

De La Cuesta, M. y Valor, C. (2003). Responsabilidad social de la empresa. Concepto, medición y desarrollo en España. Boletín Económico De ICE, Información Comercial Española, 2755, 7-20.

Decker, O.S. (2004). Corporate social responsibility and structural change in financial services. Managerial Auditing Journal, 19(6), 712-728.

Frederick, W.C. (1960). The growing concern over business responsibility. California Management Review, 2(4), 54-61. 
Frederick, W.C. (1983). Corporate social responsibility in the eagan era and beyond. California Management Review, 25(3), 145-156.

Illia, L., Rodríguez, B., González, A. y Romenti, S. (2010). La comunicación de la RSC entre las 250 principales empresas europeas. Cuadernos de Información, 27, 85-96.

Jones, T.M. (1980). Corporate social responsibility revisited, redefined. California Management Review, 22(3), 59-67.

Kerlinger, F. N. (1986). Foundations of behavioral research. ( $3^{\mathrm{a} e d .) . ~ N e w ~ Y o r k: ~ H o l t, ~ R i n e h a r t ~ y ~ W i n s t o n . ~}$

Kok, P., Van Der Wiele, T., Mckenna, R. y Brown, A. (2001). A corporate social responsibility audit within a quality management framework. Journal of Business Ethics, 31(4), 285-297.

Maignan, I. y Ferrell, O.C. (2004). Corporate social responsibility and marketing: An integrative framework. Journal of the Academy of Marketing Science, 32(1), 3-19.

Mediaresponsables y Dircom. (2010). La aplicación de las herramientas de Comunicación a la RSE. Barcelona.

Mögele, B. y Tropp, J. (2010). The emergence of CSR as an advertising topic: A longitudinal study of German CSR advertisements. Journal of Marketing Communications, 16(3), 163-181.

Mohr, L.A., Webb, D.J. y Harris, K.E. (2001). Do consumers expect companies to be socially responsible? The impact of corporate social responsibility on buying behavior. The Journal of Consumer Affairs, 35(1), 45-72.
Nestlé (2014, 4 de enero). Nestlé tops leading sustainability indices. Disponible en:

http://www.nestle.com/media/newsandfeatures/nestle-topsleading-sustainability-indices

Panwar, R., Rinne, T., Hansen, E. y Juslin, H. (2006). Corporate responsibility: Balancing economic, environmental, and social issues in the forest products industry. Forest Products Journal, 56(2), 4-12.

Podnar, K. (2008). Communicating Corporate Social Responsibility. Journal of Marketing Communications, 14(2), 75-81.

Smith, N.C. (2003). Corporate social responsibility: Whether or how?. California Management Review, 45(4), 52-76.

Unilever (2014, 4 de enero). Awards and recognition. Disponible en:

http://www.unilever.com/sustainable-living/ourapproach/ awards/

Van Marrewijk, M. (2003). Concepts and definitions of CSR and corporate sustainability: Between agency and communion. Journal of Business Ethics, 44(2/3), 95-105.

Waldman, D.A., De Luque, M.S., Washburn, N. y House, R.J. (2006). Cultural and leadership predictors of corporate social responsibility values of top management: A GLOBE study of 15 countries. Journal of International Business Studies, 37(6), 823-837.

Watson, M. y Mackay, J. (2003). Auditing for the environment. Managerial Auditing Journal, 18(8), 625-630. 
